\title{
Physics and Electronics Simulations of the Large Pixel Detector at EuXFEL
}

\author{
Ashley Joy, Marcus French, Matthew Hart, Paul Seller, Matthew Wing
}

\begin{abstract}
A simulation of the Large Pixel Detector (LPD), an $\mathrm{X}$-ray pixel detector at EuXFEL, is presented. HPAD Output Response fUnction Simulator (HORUS) was written in IDL to model the Adaptive Gain Integrating Pixel Detector (AGIPD), another X-ray pixel detector at EuXFEL; HORUS_LPD is an adaptation of the code to model LPD. While the original program was intended and designed to be modular, the large differences in the architecture of the Front End Modules (FEMs) in AGIPD and LPD required most of the code to be rewritten, particularly the triple amplification used by LPD. The program simulates from the absorption of photons in the silicon to the final readout image and all sources of noise expected in the real system. The simulation is written in Interactive Data Language (IDL), a programming language specifically intended for working with images or large arrays, functionality that is utilized by HORUS and HORUS_LPD. The simulation was tested using example data and an analysis of the noise in LPD was conducted.
\end{abstract}

\section{INTRODUCTION}

W RITTEN in IDL, HORUS_LPD is a software tool that simulates the physics and electronics response of LPD, a 1 Megapixel X-Ray camera under development at Rutherford Appleton Laboratory (RAL) for use at the European XFEL.

From an array describing the photons incident on the detector, HORUS_LPD generates the complete picture that will be delivered to the user, including physics simulations of the photons, interactions and electron cloud spread, then electronic simulations of amplification, storage, digitization and image reconstruction.

The program was developed from HORUS [1], the tool built to simulate LPD's sister detector AGIPD. The physics model from HORUS was retained, though expanded in some areas, the electronics model was rewritten to take account of differences in the layout of the ASIC chips and modules.

LPD is a 2D, 1 Megapixel X-ray detector being designed and built at Rutherford Appleton Laboratory for use at XFEL. 256 modules in 16 super modules make up the detector, each super module having a single connection to the clock \& control system [2] for turning the modules on and off, and a single connection to the data acquisition system for data read out. [3]. Eight ASIC chips bonded to a single 500 micrometer thick piece of silicon form a module, creating a $36 \times 128$ grid of pixels, each 500 micrometers cubed.

The detector was specifically designed to work at the European XFEL, which produces high brilliance laser light

Manuscript received November 16, 2012.

Ashley Joy and Matthew Wing are with University College London, London, United Kingdom.

Marcus French, Matthew Hart and Paul Seller are with Rutherford Appleton Laboratory, Didcot, United Kingdom. down to $0.05 \mathrm{~nm}$ wavelengths. In addition to each pixel using either a $5 \mathrm{pF}$ or $50 \mathrm{pF}$ capacitor, which can be set by the user in advance of the experiment, LPD uses a triple gain system, where the signal is split three ways, with each signal amplified by a different rate and stored. This system gives LPD a dynamic range of 100,000 pixels at $12 \mathrm{keV}$, or a $1.2 \mathrm{GeV}$ total signal.

XFEL will operate at a peak pulse rate of $4.5 \mathrm{MHz}$ for $0.6 \mathrm{~ms}$ during the bunch trains, with a $99.4 \mathrm{~ms}$ gap between each train. LPD will be able to store up to 512 images from each bunch train, utilizing a veto signal from the clock \& control system to choose which images to discard, the detector storing each image during the trains and reading out in the intervening gap.

\section{Simulation Development}

HORUS_LPD was developed from HORUS [1], a physics and electronics simulation of the AGIPD detector, one of the other detectors being built for use at XFEL, written by Guillaume Potdevin and Julian Becker. Though written in modular components and intended to be ported to other detector designs, enough of HORUS required re-writing that most of the HORUS_LPD code is no longer compatible with that from HORUS.

The simulation can be broken into two parts, the physics and the electronics simulation. The Physics model begins at the facing edge of the silicon, for each photon incident on a pixel it calculates a random position within the pixel and an interaction distance. Accounting for the angle of the incoming pixel the photon is discarded if it has left the silicon completely, if not then a single point of interaction is assumed and the position of this is calculated from the interaction length and the angle of the photon. From the point of interaction an electron cloud and its spread is calculated from the point of interaction and the amount of charge landing in each pixel, including charge sharing between pixels, is calculated. The angle of the photon is calculated based on the distance from the sample, provided by the user, and the position of the detector. LPD has been designed to be placed either centrally along the beam line, above it, or above it and tilted forwards, the simulation of these layouts was added with HORUS_LPD.

The electronics simulation takes over after the physics simulation calculates the charge deposited on each pixel. The signal in each pixel is copied to three arrays, each array is then multiplied by a different gain value, corresponding to the 1, 10 and 100 amplification factors used by LPD. The effects of storage in capacitors, the digitization of the signal and the 
selection of the best of the three gain signals, per pixel, by the FEM is then simulated. Gaussian distributed noise can be added at the amplification, storage, digitization and signal selection stages, the last of these is used in replacement of the other three when the user wishes to use, or only knows, the noise of the entire ASIC. The image is then reconstructed and given to the output. The ability to use either a $5 \mathrm{pF}$ or $50 \mathrm{pF}$ capacitor in each pixel is not simulated, because this has the effect of changing the gain and noise parameters this is currently simulated by running the simulation twice, with the entire detector se to either $5 \mathrm{pF}$ or $50 \mathrm{pF}$.

\section{Testing}

To test the simulation an example image was created as an input, shown in Fig. 1. This image was based upon simulated photon scatter used in a K. Haldrup, H. Lemke and $M$. Nielsen[4]. The intensity of the image was scaled such that it would test the entire dynamic range of the detector.

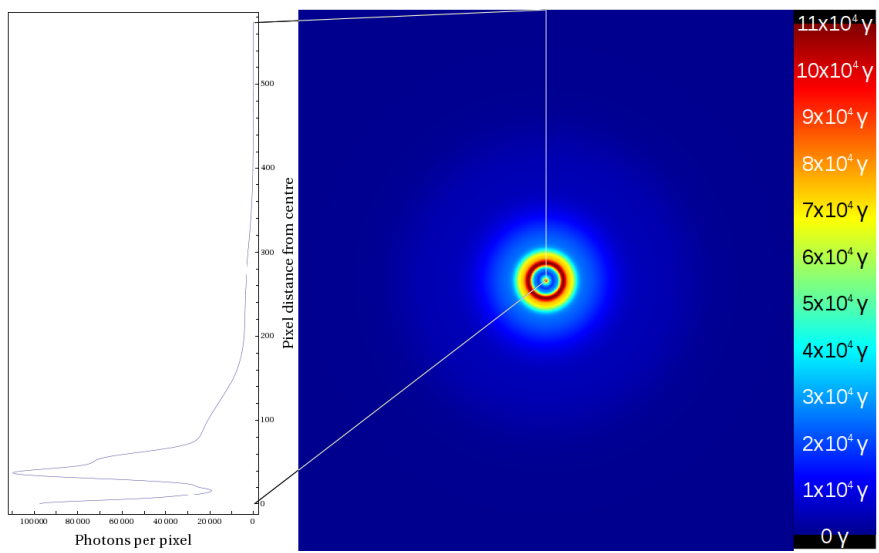

Fig. 1: The input image used in the testing of HORUS_LPD, together with the intensity line graph of the cross section.
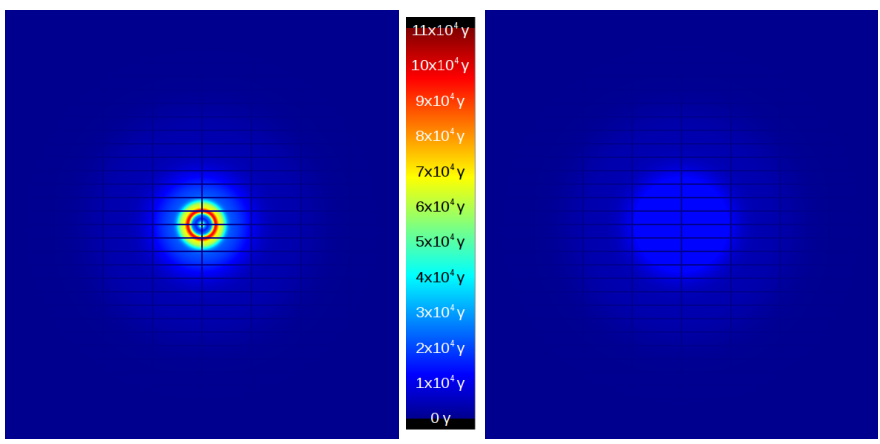

Fig. 2: The output images produced by HORUS_LPD, set at $50 \mathrm{pF}$ and $5 \mathrm{pF}$.

Because of the inefficiency of silicon at the energy tested, $12.4 \mathrm{keV}$, there is a noticeable drop in intensity across the entire output image shown in Fig. 2, in addition the blank areas between modules are visible as they are displayed here as areas with zero intensity. The right hand image of Fig. 2 lacks features in the centre of the image, since here the input exceeded the range of the $5 \mathrm{pF}$ setting. The effects of noise are not visible at this intensity range.

\section{NOISE IN LPD}

With simulated data now on hand the effect of noise on the final output image was calculated. This was done by scaling the intensity of the input image down by the inefficiency of the silicon at $12.4 \mathrm{keV}$ and comparing it to the output image to produce a noise image.
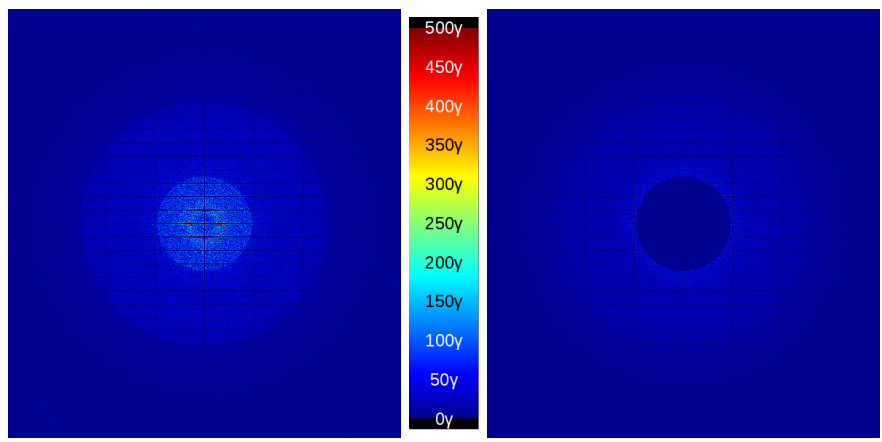

Fig. 3: The noise on the images provided by HORUS_LPD, set at $50 \mathrm{pF}$ and $5 \mathrm{pF}$.

Though the areas where gains switch are clearly visible on the $50 \mathrm{pF}$ image as steps in noise intensity, they are far harder to spot on the $5 \mathrm{pF}$ image, where photon counting statistics dominates in most pixels. The centre of the $5 \mathrm{pF}$ image has been left blank because the centre of the image exceeded the dynamic range of the $5 \mathrm{pF}$ setting, thus the 'noise' there is meaningless.

The data from these noise images was compiled together and drawn up as a histogram, shown in Fig. 4, that graphically demonstrates the effect of counting statistics noise and electronics noise across the majority of the dynamic range.

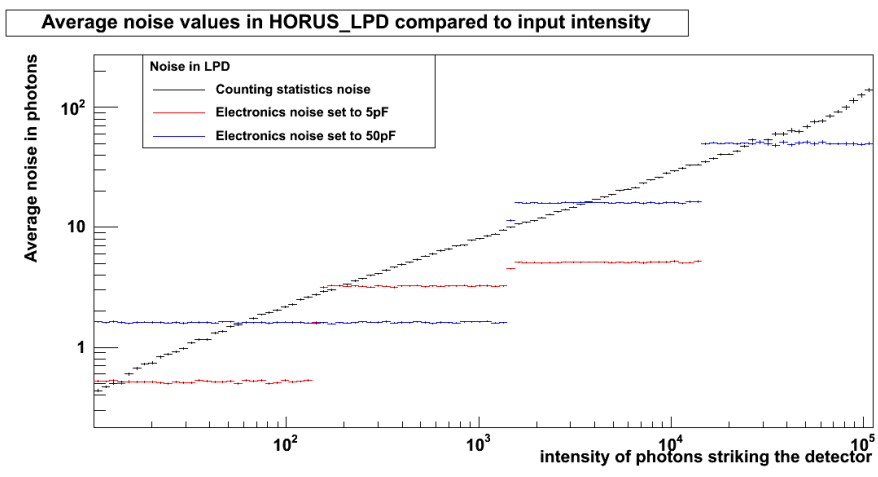

Fig. 4: Average noise values due to counting statistics and electronic noise across the LPD dynamic range.

\section{Future Replacement}

A Geant4 model of LPD is currently under development, which will be used to model radiation dosage levels and the rate of fluorescence in the materials used to make the modules. In particular this will be used to analyse the effects of operating LPD at the higher energy range of XFEL, between $12 \mathrm{keV}$ and $24 \mathrm{keV}$.

A great deal of experience has been gained while creating and working with HORUS_LPD, however while a detector 
simulation like HORUS is desired at EuXFEL it has been decided that a total replacement of HORUS and HORUS_LPD would be a better long term solution and this is now in the planning stages. The replacement simulation will be written in C++ integrated into the Karabo XFEL suite software package at EuXFEL. This will model all three detectors being built specifically for XFEL as well as any other purchased commercially, because of this the replacement simulation will be written with the intention of being easy to adapt to other detectors.

\section{CONCLUSION}

HORUS_LPD is a simulation of the LPD detector being built for use at EuXFEL. The simulation covered the physics of photon interaction and charge sharing within the silicon layer of the detector as well as the electronics of the ASIC chips, Front end modules and train builder. The simulation has been tested with example data and used by the group building LPD to better understand their electronics. A replacement for HORUS_LPD improving upon it and written in an open programming language is currently entering development.

\section{REFERENCES}

[1] G. Potdevin, U. Trunk and H. Graafsma, "HORUS, an HPAD X-ray detector simulation program", 2009 JINST 4 P09010

[2] E. Motuk, M. Postranecky, M. Warren and M. Wing, 2011 JINST 7 (2012) $\mathrm{C} 01062$

[3] J. Coughlan, S. Cook, C. Day, R. Halsall and S. Taghavi, 2011 JINST 6 C12057

[4] K. Haldrup, H. Lemke, M. Nielsen, "Considerations of S/N in solution scattering studies using a detector with noisy readout" 